\title{
Different Behavior of Bioresorbable Vascular Scaffold and Metallic Stent With Positive Vessel Remodeling
}

\author{
Satoru Mitomo, MD; Damiano Regazzoli, MD; Luciano Candilio, MD; \\ Akihito Tanaka, MD; Azeem Latib, MD; Antonio Colombo, MD
}
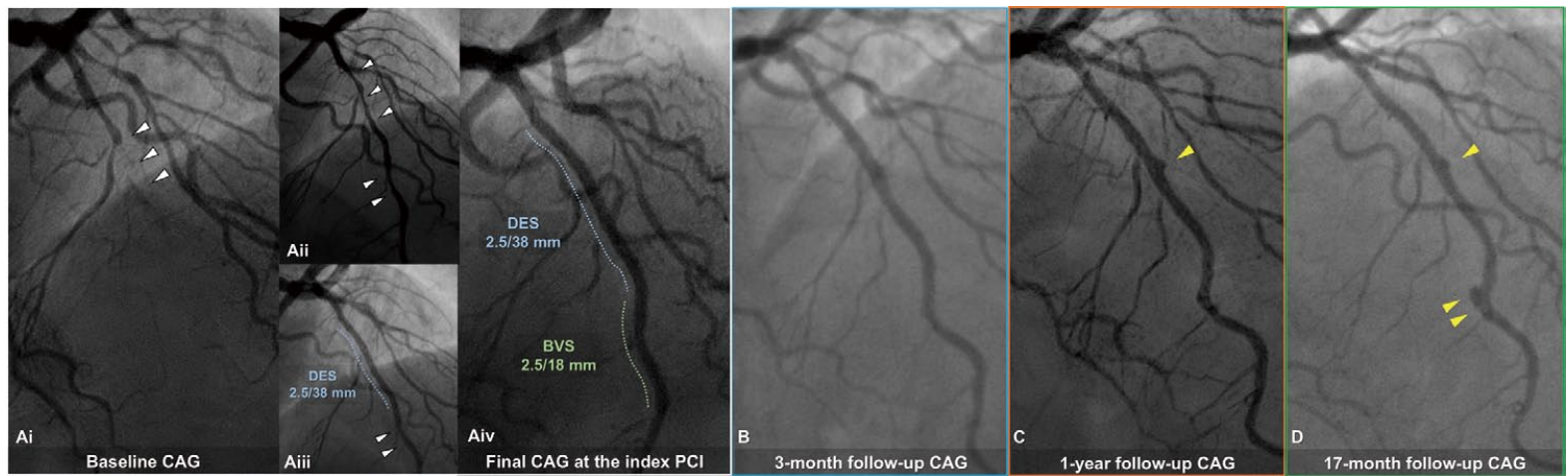

Figure 1. Serial coronary angiography (CAG) changes in a coronary aneurysm. (Ai) Baseline CAG at presentation (arrowheads, occluded left anterior descending artery [LAD]). (Aii) After recanalization of the occluded LAD with wire crossing and nitrate injection (arrowheads, proximal culprit lesion and distal stenotic lesion). (Aiii) After drug-eluting stent (DES; Xience Alpine 2.5/38 $\mathrm{mm}$ ) implantation for the proximal lesion (blue dashed line, lesion treated with DES; arrowheads, distal residual lesion). (Aiv) After bioresorbable vascular scaffold (BVS; Absorb BVS 2.5/18 mm) implantation for the distal lesion (dashed lines, lesion treated with [blue] DES and [green] BVS). (B-D) Follow-up CAG at (B) 3 months; (C) 1 year (arrowhead, aneurysmal change in the DEStreated segment); and (D) 17 months (arrowheads, aneurysmal changes in the segment treated with [single arrowhead] DES and [double arrowhead] BVS).

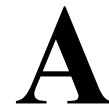
54-year-old woman presented to her local hospital with an anterior myocardial infarction and was treated with a 2.5/38-mm drug-eluting stent (DES; Xience Alpine, Abbott Vascular, Santa Clara, CA, USA) to the occluded mid left anterior descending (LAD) artery (Figure 1Ai-Aiii). A bioresorbable vascular scaffold (Absorb BVS; $2.5 / 18 \mathrm{~mm}$, Abbott Vascular) was then implanted electively in the distal LAD (Figure 1Aiv), with excellent results confirmed at 3-month coronary angiography (CAG; Figure 1B). No intravascular imaging was performed during these 2 procedures. Dual antiplatelet therapy (DAPT) consisting of aspirin $100 \mathrm{mg} /$ day and ticagrelor $90 \mathrm{mg}$ twice/ day was given for 12 months, then switched to aspirin $100 \mathrm{mg} /$ day and clopidogrel $75 \mathrm{mg} /$ day.

One year after the procedure, the patient remained asymptomatic, on prolonged DAPT, and underwent followup CAG at San Raffaele University Hospital, Milan. No significant restenosis was seen, although there were focal aneurysmal changes in the segment previously treated with DES (Figure 1C). Further CAG 5 months later showed no obvious angiographic changes of the aneurysm, and a new aneurysm in the distal LAD at the level of the BVS (Figure 1D). On optical coherence tomography, aneurysmal changes in the mid LAD had resulted in major malapposition of the metal struts (maximum, $880 \mu \mathrm{m}$; Figure 2A-D) without evidence of thrombus. In contrast, the BVS followed the vessel wall, even in the aneurysmal segment, with good endothelialization and no malapposed struts in the whole BVS segment (Figure 2E-H). No additional intervention was performed due to the focal aspect of the malapposition and the complete endothelialization of all remaining struts, and the patient continued on prolonged DAPT.

Vessel remodeling following percutaneous coronary

Received August 7, 2017; revised manuscript received September 12, 2017; accepted September 19, 2017; released online October 13, 2017 Time for primary review: 14 days

Unit of Cardiovascular Interventions, IRCCS San Raffaele Scientific Institute, Milan (S.M., D.R., L.C., A.T., A.L., A.C.), Italy; The Hammersmith Hospital, Imperial College London, London (L.C.), UK; and Vita-Salute San Raffaele University, Milan (A.C.), Italy

Mailing address: Antonio Colombo, MD, IRCCS San Raffaele Scientific Institute, Via Olgettina 60, 20132 Milan, Italy. E-mail: info@emocolumbus.it

ISSN-1346-9843 All rights are reserved to the Japanese Circulation Society. For permissions, please e-mail: cj@j-circ.or.jp 


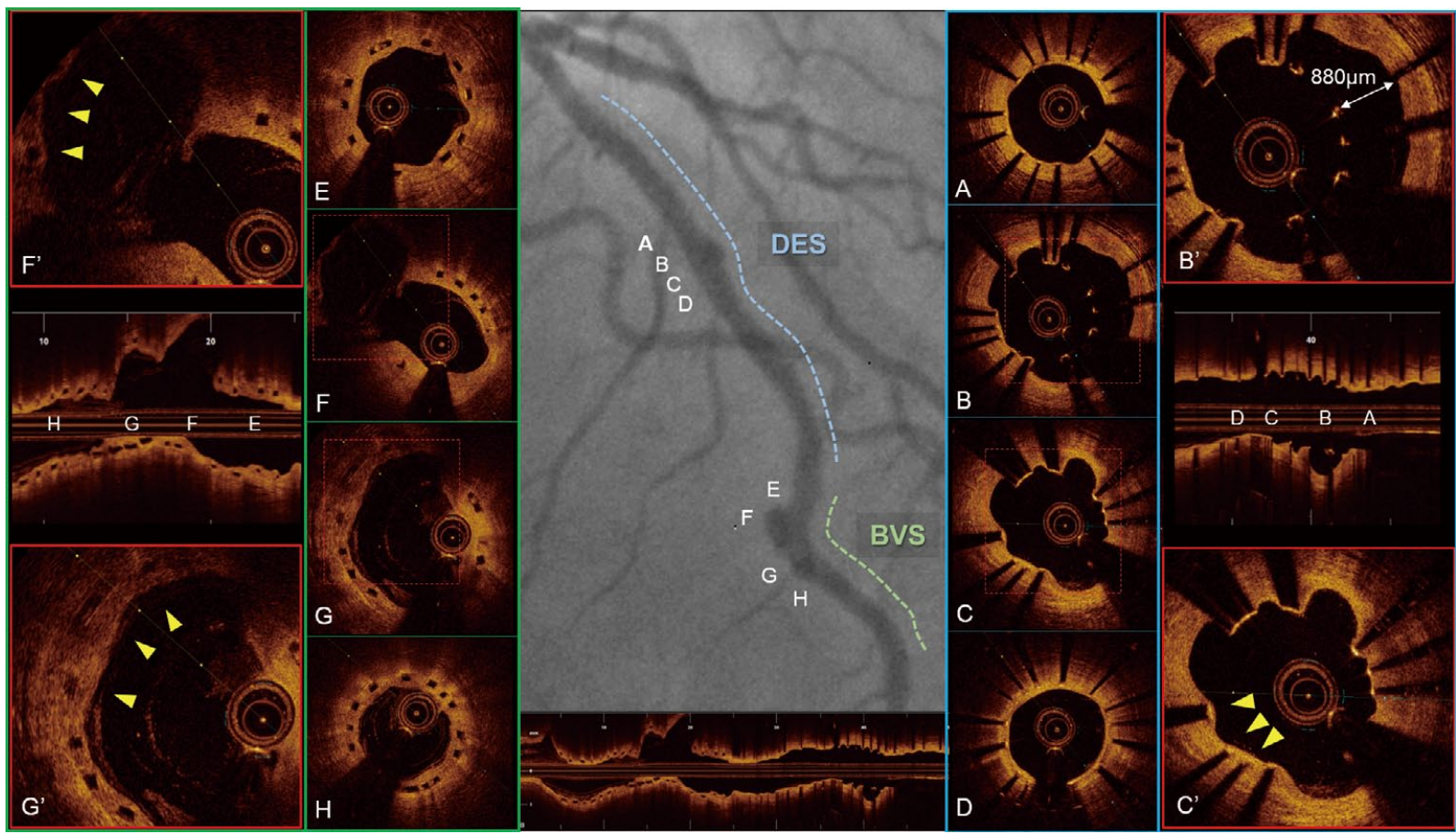

Figure 2. Optical coherence tomography (OCT) of the segments treated with (A-D) drug-eluting stent (DES) and (E-H) bioresorbable vascular scaffold (BVS). (A,D) Proximal and distal to the lesion with aneurysmal changes (all DES struts are apposed with complete endothelialization). (B,C) Segment with aneurysmal changes; (B',C') magnification of the red dashed line areas in (B,C). (B') (maximum malapposition): stent diameter, 2.33/2.43 mm (mean, 2.38 mm); lumen diameter, 2.84/3.41 mm (mean, $3.12 \mathrm{~mm}$ ). (C') Arrowheads, partial endothelialization of struts at the segment with aneurysmal change. (E,H) Proximal and distal to the segment with aneurysmal changes (all scaffolds are endothelialized). (F,G) Segment with aneurysmal changes; (F', G') magnification of the red dashed line areas in $(\mathbf{F}, \mathbf{G})$. Arrowheads, scaffold following vessel wall with aneurysmal changes.

intervention is unpredictable, and aneurysm formation can lead to late malapposition, ${ }^{1}$ especially with metallic stents, due to their rigidity, thereby resulting in higher risk of stent thrombosis. ${ }^{2}$ Conversely, if the BVS is well embedded in the vessel wall and endothelialized, it is likely to follow the vessel wall even in the context of aneurysmal changes. This case illustrates the different behavior of BVS during the DES resorption process in this type of lesion.

\section{Disclosures}

The authors declare no conflict of interest. A.L. is a consultant for
Medtronic; and has received honoraria from Abbott Vascular. All other authors have reported that they have no relationships relevant to the contents of this paper to disclose.

\section{References}

1. Kang SJ, Mintz GS, Park DW, Lee SW, Kim YH, Lee CW, et al. Late and very late drug-eluting stent malapposition: Serial 2-year quantitative IVUS analysis. Circ Cardiovasc Interv 2010; 3: $335-340$.

2. Taniwaki M, Radu MD, Zaugg S, Amabile N, Garcia-Garcia $\mathrm{HM}$, Yamaji K, et al. Mechanisms of very late drug-eluting stent thrombosis assessed by optical coherence tomography. Circulation 2016; 133: 650-660. 